\title{
The Sufferings of Cancer Patients Infected by SARS-CoV-2
}

\author{
Zinat Mahal ${ }^{1}$ and Hasan M Zahid ${ }^{2 *}$ \\ ${ }^{1}$ Microbiology and Industrial Irradiation Division, Institute of Food and Radiation Biology, Bangladesh \\ ${ }^{2}$ Institute of Tissue Banking and Biomaterial Research, Bangladesh
}

*Corresponding author: Hasan M Zahid, Institute of Tissue Banking and Biomaterial Research, AERE, Ganakbari, Savar, Dhaka1349, Bangladesh.

To Cite This Article: Zinat Mahal, Hasan M Zahid, The Sufferings of Cancer Patients Infected by SARS-CoV-2. 2020 - 11(2). AJBSR.MS.ID.001618. DOI: 10.34297/AJBSR.2020.11.001618.

Received: 㘹 December 04, 2020; Published: 眥 December 15, 2020

\section{Opinion}

The coronavirus disease 2019 (COVID-19), caused by severe acute respiratory syndrome coronavirus-2 (SARS-CoV-2), has drawn attention in the world in early 2020. This is an infectious disease occurs by an unusual type of coronavirus, spreads easily and has a significant mortality rate. Among different types of coronaviruses, some cause mild sickness i.e., cold, sneeze while others can be more serious with severe complications. This virus spread from person to person, particularly when a COVID-19 patient delivers droplets with sneezes or coughs. The common symptoms of coronavirus include a high temperature of above $37.80 \mathrm{C}$, continuous cough, and a loss of/change in normal sense of taste or smell. In this write-up, we have tried to accumulate the currently available information on the outcome of SARS-CoV-2 infection on patients with cancer comorbidities [1].

The immune system protects body against illness and infection caused by viruses like coronavirus. Cancer and its treatment can also weaken the immune system and reduce ability to fight infections. Therefore, a cancer patient is at an increased risk of complications for COVID-19 outbreak because of weak immune systems for cancer or current treatment such as chemotherapy, radiotherapy etc. Some people with cancer are more at risk of being seriously ill if they develop COVID-19. They are called extremely vulnerable (Table 1), they must be alert to prevent from getting ill by SARS-CoV-2, be aware about the frequency of infections in the infected area.

In general, staying at home, limited contact with others outside the household, following strict social distancing with a face covering, washing hands or use hand sanitizing alcohol gel regularly, wearing a face covering shield may limit the action of SARS-CoV-2. So, if people are not in one of the above vulnerable groups, they may conquer COVID-19 following these normal regulations. However, the vulnerable groups, of course, need extra care as they suffer critical illness, in some extent they may die.

The prevalence of cancer among patients infected with SARSCoV-2 was higher than in the general population. One percent of the COVID-19 cases had a history of cancer when compared to $0.29 \%$ of the Chinese population. The most cancer patients suffer critical sickness when they become COVID-19 positive (Table 2) [2].

Table 1: The factors associated with the extremely vulnerable cancer patients.

\begin{tabular}{|c|r|}
\hline \multirow{4}{*}{$\begin{array}{l}\text { Extremely vulnera- } \\
\text { ble cancer patients }\end{array}$} & With cancers of the blood or bone marrow such as leukemia, lymphoma or myeloma who are at any stage of treatment \\
\cline { 2 - 3 } & getting immunotherapy or other antibody treatments for cancer \\
\cline { 2 - 3 } & Getting any targeted cancer treatments i. e., protein kinase inhibitors \\
\cline { 2 - 3 } & WHO conducted bone marrow or stem cell transplants in the last six months, or who are still taking immune-suppression drugs \\
\hline
\end{tabular}


Table 2: The effect of SARS-CoV-2 infection in cancer co-morbidities.

\begin{tabular}{|l|c|}
\hline \multirow{2}{*}{ Cancer patients suffering severe illness while infected with SARS-CoV-2 } & Cancer/affected organs \\
\cline { 2 - 2 } & Lung \\
\cline { 2 - 2 } & Colorectal \\
\cline { 2 - 2 } & Breast \\
\cline { 2 - 2 } & Bladder \\
\cline { 2 - 2 } & Pymphoma \\
\cline { 2 - 2 } & Penallary thyroid cancer carcinoma \\
\hline
\end{tabular}

Though WHO set up several initiatives to control SARS-CoV-2 outbreak, researchers are working hard over the past year to develop COVID-19 vaccines. Several vaccine trials are in progress worldwide and some of the results have now been published. The results are satisfying. However, it is not established enough about how well vaccines will work particularly in the management of cancer patients.

In general, it is understandable that the recent outbreak significantly affects cancer patients. The association between malignant co-morbidity and mortality from COVID-19 is clear.

In some cases, cancer patients are prone to develop psychiatric disease including anxiety and depression etc. [3]. We all including health care professionals should keep report any cases of cancer patients infected with SARS-CoV-2, their management, and the outcome in order to further understanding of this complexity.
We believe that as more data becomes available, it is going to be necessary to publish detailed guidelines on how to approach this unique clinical challenge.

\section{References}

1. Adhikari SP, Meng S, Wu YJ, Mao YP, Ye RX, et al. (2020) Epidemiology, causes, clinical manifestation and diagnosis, prevention and control of coronavirus disease (COVID- 19) during the early outbreak period: a scoping review. Infect Dis Poverty 9(1): 29.

2. Liang W, Guan W, Chen R, Wei W, Jianfu L, et al. (2020) Cancer patients in SARS-CoV-2 infection: a nationwide analysis in China. Lancet Oncol 21(3): 335-337.

3. Kotronoulas G, Papadopoulou C, Burns-Cunningham K, Simpson M, Maguire R (2017) A systematic review of the supportive care needs of people living with and beyond cancer of the colon and/or rectum. Eur J Oncol Nurs 29: 60-70. 